\title{
Reflexión sobre los propósitos de la sociedad y la educación actual ${ }^{1}$
}

\author{
Luis Herrera Vásquez*
}

\begin{abstract}
Resumen
En el presente artículo se desarrolla un análisis sobre los lineamientos sociales inmersos en el sistema neoliberal, que determinan los propósitos de nuestra educación actual y, finalmente, una reflexión de los objetivos que debiera tener. Se revisan autores como Noam Chomsky, Slavoj Zizek, Guy Debord, Jean Baudrillard, que entregan un panorama bastante crítico sobre el ser humano post moderno. Además, se analiza el estado presente de nuestra educación, mediante sus características y resultados evaluativos, para, finalmente, desde una perspectiva que se apoya en Paulo Freire, Torres y Chomsky, plantear la posibilidad de desarrollar una educación transformadora, integral y crítica.
\end{abstract}

Palabras clave: Educación, Economía neoliberal, Currículum oculto, sociedad.

\section{Reflections on the purposes of society and education today}

\section{ABSTRACT}

This paper analyzes the social guidelines imbedded in the neoliberal system, which determine the purposes of our current education, and reflects on the purposes it should have. It reviews authors as Noam Chomsky, Slavoj Zizek, Guy Debord and Jean Baudrillard, who deliver a highly critical view about the post-modern human. Furthermore, it looks upon the present situation of our education through its features and evaluation results to finally suggest the possibility of developing a transformative, comprehensive and critical education, from a perspective supported by Paulo Freire, Torres and Chomsky.

Key words: Education, neoliberal economy, hidden curriculum, society.

I Parte del proyecto de REDISEÑO CURRICULAR DE PEDAGOGÍA EN CASTELLANO 20I4, en la etapa previa de análisis contextual.

* Magíster en docencia universitaria, Universidad Autónoma de Chile. Chileno. e-mail:

lherrerav@uautonoma.cl 


\section{Introducción}

Tener conciencia clara de que las respuestas factibles de construir ante el qué, el cómo, el cuándo, el dónde educar apuntan necesariamente a la práctica y se relacionan directamente con lo explícito en el currículum declarado de una institución. No obstante, dichas preguntas no manifiestan los propósitos que posee la educación institucional o social-, es decir, ¿para qué educar?, pregunta evidente, pero ausente de gran parte del debate pedagógico, principalmente porque la educación se ha orientado a responder a las necesidades de la sociedad y no, como varios autores vienen planteando hace décadas, a transformarla. Esto, probablemente ocurre porque el sistema de mercado y las organizaciones gubernamentales no se cuestionan las falencias de la sociedad actual, sino que buscan, indefectiblemente, subirse al carro de la globalización y el desarrollo.

Por lo tanto, no siempre coincidimos con claridad en el "ipara qué educar?”. Podríamos llegar a ciertos consensos, pero muchos de ellos no pasan de ciertos ensayos que no discriminan entre la realidad y lo políticamente correcto de declarar. Inclusive, no es poco común oúr de parte de muchos actores, aun profesores, que la educación se trata de restricciones, normas, modelamientos, competencia y exitismo; en desmedro de un desarrollo íntegro del complejo ser humano, y en favor de un sistema de oferta-demanda:

Nuestro sistema educativo tiene una larga tradición de abandono por parte de los poderes públicos y es ahora, en los momentos en que los desajustes entre lo que las instituciones escolares ofrecen y lo que la sociedad demanda son más chirriantes, cuando es necesario realizar diagnósticos más precisos que faciliten la comprensión de los porqués del estado actual de la educación y, de este modo, tomar las medidas más convenientes para su corrección (Torres. I998: 9).

De fondo, entonces, se sostiene que:

(...) todos los sistemas educativos se mantienen y justifican sobre la base de líneas de argumentación que tienden a oscilar entre dos polos discursivos, por una parte las que defienden que la educación es una de las vías privilegiadas para paliar 
y corregir las disfunciones de las que se resiente el modelo socioeconómico y cultural vigente y, por otra, las que sostienen que las instituciones educativas pueden ejercer un papel decisivo en la transformación y el cambio de los modelos de sociedad de los que venimos participando (Torres, I998: I2).

En síntesis, en la actualidad el “¿Para qué educar?” no pareciera responder a lo que se declara con tanto énfasis: una educación integral, que apunte a la autonomía, la creatividad y la identidad. Es necesario, entonces, discutir sobre qué somos hoy como sociedad inserta en el sistema neoliberal, de qué manera aquello determina nuestra educación actual y qué objetivos debiéramos plantearnos frente a ella.

\section{El sistema neoliberal y la educación actual}

En su libro "Piratas y emperadores", Noam Chomsky señala lo siguiente:

El filósofo John Dolan observa: "La gente no carecerá del valor para expresar pensamientos fuera del alcance permitido, sino que se verá privada de la capacidad de pensar tales cosas". Esa es la cuestión esencial, la fuerza impulsora de los ingenieros del consenso democrático (Chomsky, 2003: 56).

Es muy pertinente detenerse en la relectura del enunciado de Chomsky, pues señala que la gente no carecerá del valor para expresar lo que piensa, es decir, hará uso de la libertad de expresión -siempre en un contexto democrático- para expresarse fuera del alcance permitido, pero se verá privada de reflexionar, analizar, llegar a tener las capacidades para pensar fuera de dicho alcance, gracias a la planificada acción de los ingenieros del consenso democrático. Ahora bien, ¿quiénes son tales ingenieros? ¿Los gobiernos? ¿El poder económico? ¿El profesorado? En cierta forma, responde Chomsky, todo un conjunto de actores: (...) "el control del pensamiento es una industria próspera, ciertamente indispensable en una sociedad libre basada en el principio de decisión de la elite y en el respaldo o pasividad del público”. (Chomsky, 2003: 58). 
Si consideramos la planificación de los "ingenieros del consenso democrático”, que indica el intelectual norteamericano, se puede colegir que los objetivos subyacentes a tal planteamiento, nos llevan a autores como Guy Debord, quien indica que:

La primera fase de la dominación de la economía sobre la vida social había implicado en la definición de toda realización humana una evidente degradación del ser en el tener. La fase presente de la ocupación total de la vida social por los resultados acumulados de la economía conduce a un deslizamiento generalizado del tener al parecer, donde todo "tener" efectivo debe extraer su prestigio inmediato y su función última. (Debord, 1967: 5).

La crítica al "parecer" que realiza Debord, expone el traslado desde una sociedad centrada en lo humano hacia una sociedad centrada en la posesión privada y, actualmente, centrada en el prestigio de la posesión. Pareciera que el consumo y la imagen, indica Debord, se han tomado los propósitos de todas las acciones sociales. Lo que tengo y proyecto al otro ha alcanzado ribetes de tal trascendencia que el carácter individual, autónomo, creativo y único del ser humano se ha perdido.

A su vez, Jean Baudrillard, en la misma sintonía, especifica que se ha perdido el "Ser" auténtico en favor de una "imagen" simulada:

Las fases sucesivas de la imagen serían éstas:

- es el reflejo de una realidad profunda

- enmascara y desnaturaliza una realidad profunda

- enmascara la ausencia de realidad profunda

- no tiene nada que ver con ningún tipo de realidad, es ya su propio y puro simulacro. (Baudrillard, I993: I4).

En el mundo de la imagen, se comprende en el autor francés, hemos perdido toda noción de la realidad. Si en un principio la imagen surge como reflejo de la realidad profunda, esto se desvirtúa de tal manera que se pierde toda relación y pasa a ser un mero simulacro; ya sea matizada por el consumo, ya sea influenciada por el espectáculo y los medios de comunicación. Este trastorno de la simulación es ejemplificado con máxima claridad por Baudrillard: 
(...) se ha prohibido visitar las grutas de Lascaux, pero se ha construido una réplica exacta a 500 metros del lugar para que todos puedan verlas (se echa un vistazo por la mirilla a la gruta auténtica y después se visita la reproducción). Es posible que incluso el recuerdo mismo de las grutas originales se difumine en el espíritu de las generaciones futuras, pero no existe ya desde ahora diferencia alguna, el desdoblamiento basta para reducir a ambas al ámbito de lo artificial (Baudrillard. 1993: 20).

Esta crisis del "ser", pasando de una lógica humana a una lógica del "tener" y de la "imagen", responde a una lógica de mercado que amerita ser revisada, reflexionada y discutida como toda actividad que comprometa a nuestra sociedad. De una manera bastante radical, Slavoj Zizek ha configurado una postura aun más extrema sobre nuestra sociedad y la economía:

La gran novedad de la era pospolítica actual -la era del "fin de las ideologías" - es la despolitización radical de la esfera de la economía: el modo en que la economía funciona (la necesidad de recortar el gasto social, etc.) es aceptado como un simple dato del estado de cosas objetivo. Sin embargo, en la medida en que esta despolitización fundamental de la esfera económica sea aceptada, todas las discusiones sobre la ciudadanía activa y sobre los debates públicos de donde deberían surgir las decisiones colectivas seguirán limitadas a cuestiones "culturales" de diferencias religiosas, sexuales o étnicas -es decir, diferencias de estilos de vida- y no tendrán incidencia real en el nivel donde se toman las decisiones de largo plazo que nos afectan a todos. (...) ese socavamiento responde directamente a la despolitización de la economía, a la aceptación común del Capital y de los mecanismos del mercado como herramientas/procedimientos neutros que deben ser explotados (Zizek, I999: 2).

Señala Zizek que el mercado actual -aparentemente no ideologizado-, por supuesto que representa una ideología y en tal plano de las ideas, debiera discutirse, argumentarse y pensarse, de tal forma que se puedan generar los cambios pertinentes para no acabar con el planeta y desarrollar una sociedad más comprometida con las mejoras sociales. Si la economía es entendida como un "estado de cosas objetivo", en correspondencia con el tránsito del "ser" hacia 
el "tener" y la supremacía de la imagen, entonces, definitivamente, la educación sólo debiera responder al estado socioeconómico actual y, naturalmente, privados de la capacidad de pensar en ajustes, cambios y mejoras de nuestra sociedad.

En este nuevo escenario, claramente los medios de comunicación juegan un rol fundamental a la hora de comprender al ser humano no como un ciudadano, sino como un consumidor individual:

En el periodo moderno, los medios y la televisión son los que presentan una imagen de la vida tal como habría que vivirla según el punto de vista de los que mandan. Todos deberíamos ser consumidores felices, y consumir lo más posible. Pero a nadie le interesa, claro, si tienes que luchar en el trabajo; tus problemas existenciales, eso queda para ti al final del programa.

Y todo esto lleva intercalados, además, montones y montones de anuncios publicitarios que procuran convencer, sobre todo a los jóvenes, de que lo mejor que pueden hacer es gastar hasta el último centavo en comprar calzado de tenis de doscientos dólares, o lo que sea, porque así es como hay que vivir la vida (Chomsky, I994: 57).

En un panorama mundial de tales características y una sociedad chilena que se desarrolla muy alineada con tales preceptos, nuestra educación formal se ha configurado en respuesta a los requerimientos del mundo globalizado en detrimento de un fomento identitario y local. De tal manera, los estudiantes de los colegios chilenos han interiorizado un sentido del "¿Para qué educar?" muy poco coherente con lo que declaramos, probablemente porque lo que declaramos, no es tan coherente con nuestro actuar como agentes educativos:

Las deficiencias curriculares son otra característica generalizada en América Latina. Anotan los investigadores de la UNESCO, que los resultados de la investigación sobre los procesos de enseñanza coinciden en que "no se dan oportunidades para: reflexionar sistemáticamente; usar el conocimiento previo que tienen los alumnos sobre el tema, relacionarlo con el contexto local, discutir en grupos pequeños sobre los diversos puntos de vista y compartir las reflexiones pertinentes con la familia y la comunidad. Los currículos tampoco 
utilizan el "saber popular" ni la tradición oral de la comunidad" (Chomsky y Dieterich, I996: II7).

Según un estudio realizado por el Centro de investigación en estructura social de la Universidad de Chile, titulado "7 fenómenos sobre educación y desigualdad en Chile” (20I I), la educación para los alumnos significa sólo tres cosas: capacitación laboral, educación de la vida para afrontar las dificultades de la existencia o "buena educación" en términos de modales y criterios éticos. En otro apartado del estudio, los alumnos indican, en los propósitos de la educación, el respetar la jerarquía. Es decir, la percepción general del estudiante sobre los propósitos de su educación apuntaría a prepararse para el trabajo, enfrentar los problemas de la vida y mantenerse dentro de los marcos normativos de la sociedad. Por lo tanto, en el día a día de la educación formal, se ha ausentado la creatividad, la libertad, la autonomía y el ser un real aporte a la sociedad o, por lo menos, aquello no es percibido por los alumnos.

La situación es aún más crítica si le agregamos al significado que los estudiantes le otorgan a la educación, los niveles de desigualdad que se presentan en el modelo educativo chileno, en cuanto a segregación social y la manera en que el modelo permite la supremacía de grupos de privilegio:

(...) los resultados señalan en general que los países con calificaciones más altas tienen bajos niveles de segregación debida al status socioeconómico. Los resultados también indican que muchos de los países con sistemas escolares selectivos tienen un desempeño relativamente alto en el nivel del $4^{\circ}$ grado, antes de que los niños se vean segregados en diferentes escuelas y programas escolares, pero luego se quedan muy atrás de las pautas internacionales en los niveles más altos de educación (Wiilms, 2006, cit. en Atria. 2007: 26).

No obstante, este problema ha sido bastante estudiado y han surgido múltiples vías y argumentos para avanzar, a pesar de la segregación escolar, aunque los resultados nacionales e internacionales sigan demostrando lo contrario. Pues mientras siga existiendo la "defensa del derecho del privilegiado a transmitir su privilegio” (Atria, 2007: 39) 
entre los suyos, es decir, que "los ingenieros del consenso democrático" sigan interpretando la libertad de enseñanza como la libertad de los establecimientos de seleccionar y segregar alumnos, la educación se traducirá en pésimos resultados asociados al nivel socioeconómico:

De los estudiantes que provienen de hogares de ingresos inferiores a $\$ 278.000$ el $49 \%$ obtuvo menos de 450 en la PAA; de los alumnos que provienen de hogares con ingresos superiores a $\$ 2.500 .000$ sólo el $8 \%$ obtuvo menos de 450 puntos; de los alumnos que egresaron de la enseñanza media de colegios pagados [... I de cada 2 obtiene más de 600 puntos en la parte matemática de la PAA. En el sector particular subvencionado I de cada 15 y en el municipal I de cada 24 [...]; de los alumnos que rinden la prueba SIMCE en $8^{\circ}$ básico $[\ldots]$ en el sector pagado, I de cada 2 obtienen más de 300 puntos en la parte matemática. En el sector particular subvencionado I de cada 5 y en el municipal I de cada I0 (Fontaine, cit. en Atria, 2007: 20).

En síntesis, la educación actual ha ido fortaleciendo tendencias de modelamiento, prácticamente conductistas, que han orientado nuestras actitudes y aprendizajes a responder a los requerimientos de la sociedad, naturalmente alineadas con el consumo y el individualismo; desarrollando la percepción de que el colegio sirve para obedecer (jerarquías, normas); y en un contexto de segregación socioeconómica empoderada desde los cimientos educativos, que, dicho sea de paso, ha arrojado diferencias abismantes en los resultados de los distintos grupos sociales.

Tanto así, que el informe PISA (2009) constató que en lectura, mientras los grupos altos obtenían $5 \mathrm{I} 2$ puntos, los grupos bajos apenas 405. Esto quiere decir que, si los grupos socioeconómicos chilenos aparecieran en el ranking mundial, el grupo alto estaría en el lugar I0, por sobre Noruega, Suiza y Estados Unidos; no obstante, si apareciera el grupo bajo, estaría en el lugar 55 a nivel mundial, junto a Jordania, y superando a países tan inestables como Kirguistán, Kasajistán y Albania. De igual forma, el 52\% del grupo bajo se encuentra en el último nivel de logro en lectura, mientras apenas ocurre con el 9\% del grupo alto. Aún más preocupante, ningún integrante del grupo bajo alcanzó los dos niveles de logro 
superiores, mientras en el grupo alto sí fue alcanzado por el 5\%. Por otro lado, si bien es cierto, Chile destacó por haber subido sus puntajes del año 2000 al 2009, también llama la atención que en materia de desigualdad mantuviera las mismas brechas. Según dependencia, se obtuvieron los siguientes resultados: Municipal, 42I; Particular subvencionado, 458; y Particular, 540. Repitiendo el ejercicio anterior, si sólo participaran de la medición nuestros colegios particulares, Chile estaría en el segundo lugar a nivel mundial, sólo superado por Shangai-China.

Supongamos que la educación crítica de los estratos socioeconómicos inferiores no se deba al modelo educacional segregado y desigual, sino simplemente al determinismo cultural y al contexto familiar deprivado que rodea a tales educandos; no aparecen, de ninguna manera, soluciones más potentes a dicho problema que permitir que el privilegiado pueda transmitir su privilegio a los no privilegiados; que se combata todo tipo de segregación y determinismo educacional (lo que el alumno de niveles bajos no obtiene en su casa, que lo obtenga de sus compañeros y profesores), y que se fomente todo tipo de igualdad de oportunidades mediante la educación.

Al parecer, gran parte de nuestros proyectos de mejora no logran los resultados esperados, porque no pasan de un maquillaje superficial que se importa desde naciones desarrolladas que han logrado excelentes resultados educativos, pero en su contexto:

(...) Algunos educadores, extranjeros y locales, de las naciones emergentes, han insistido en el hecho de que la instrucción occidental fue importada negligentemente, es decir, sin consideración ni adaptación a las condiciones autóctonas, cargo éste que también puede aplicarse a otras instituciones e influencias occidentales (Tibbets et al., cit. en Peralta, 2006: 25).

De hecho, Paulo Freire señala:

Es muy importante en la teoría del currículum conocer cómo el pueblo conoce, hay que saber cómo el pueblo sabe. Hay que saber cómo el pueblo siente, cómo el pueblo piensa, cómo el pueblo habla. El lenguaje popular tiene una sintaxis, una 
estructura del pensamiento, una semántica, una significación de los significados que no puede ser, que no es igual a la nuestra, de universitarios. Y hay que conocer esto. Hay que vivir estas diferencias en las escuelas de niños populares (Cit. en Peralta, 2006: 48).

Los propósitos de la educación no sólo deben cuestionarse porque no se logran resultados o no se sube en el ranking de la competitividad educativa -porque aquello responde a la lógica del "tener" y la imagen simulada"-; tampoco porque importamos diseños extranjeros, los aplicamos en nuestros países y, así incluso, seguimos obteniendo malos resultados; los objetivos de la educación deben cuestionarse no sólo para obtener grandes logros en PISA o el SIMCE, ya que nada nos asegura que un niño que logra sobre 300 puntos en SIMCE realmente haya forjado un espíritu reflexivo, autónomo, solidario y creativo.

\section{Una reflexión propositiva de la educación}

En "Cambiar la educación para cambiar el mundo" (2007), Claudio Naranjo invita a forjar una educación para el desarrollo humano, reflexionando si estamos de acuerdo y conformes con la sociedad que somos hoy en día, problemática que es matriz de los propósitos de la educación.

Considerando lo discutido anteriormente, es necesario replantearse los objetivos de la educación. Para ello, nos detendremos nuevamente en Chomsky, quien en una entrevista realizada el 25 de enero del 2012 en Londres, en la Conferencia Aprendizaje sin Fronteras, realiza una síntesis sobre dos maneras de encarar la educación: Desde la ilustración y desde el adoctrinamiento. La primera sostiene que el objetivo más alto de la vida es crear e investigar, buscar lo significativo, es decir, el propósito de la educación es "mostrar a la gente cómo aprender por sí mismos”. La segunda, el adoctrinamiento, indica que "algunas personas tienen la idea de que, desde la infancia, los jóvenes tienen que ser colocados dentro de un marco de referencia en el que acatarán órdenes, aceptarán estructuras existentes sin cuestionar, etc.”. Chomsky opta, naturalmente, por la ilustración: 
"De hecho la Ciencia no podría progresar a menos que esté basada en la inculcación del impulso por el desafío, por el cuestionamiento de doctrinas o de la autoridad, a través de la búsqueda de alternativas o del uso de la imaginación, con el trabajo cooperativo (...)”. Frente a las evaluaciones en un modelo de "ilustración" o no enmarcado en un currículo técnico, indica el autor:

Buenas calificaciones vienen por sí solas si el tema coincide con los intereses y preocupaciones del alumno. No digo que los exámenes deban eliminarse, pueden ser una herramienta educativa útil. Pero complementaria, algo que ayude a los estudiantes a mejorar por sí mismos, o para los instructores $\mathrm{u}$ otros que necesitemos saber acerca de lo que hacemos e indicarnos lo que debemos modificar. (Chomsky, 2012).

El fracaso de nuestros planes de mejora o los diseños importados de Europa, podrían explicarse porque continuamos, insisto, centrados en el resultado final que nos expone a un ranking o una medición nacional que se centrará en separar los colegios en mejores y peores. Los buenos resultados deben llegar si nuestro trabajo pedagógico coincide con los intereses y preocupaciones del alumno, si somos respetuosos con el contexto educativo, si ayudamos a los estudiantes a mejorar por sí mismos y a pensar. Esto es como el alpinista que no logra subir a la cúspide de la montaña, porque tan enfocado en ser el primero en llegar a la cima, tropieza una y otra vez en el camino. Nuestra educación debe preocuparse más en ese camino, ya que así, con total seguridad, se llegará a la cima.

En correspondencia, Torres es igualmente concluyente:

Esta peligrosa y errónea concepción de los exámenes o evaluaciones, reducidos a ser los únicos objetivos de la escolarización, puede ser la explicación única o parcial del fracaso o las dificultades encontrados a la hora de introducir innovaciones en los contenidos y en las estrategias de enseñanza y aprendizaje en las aulas (Torres, I998: 36).

Concluye Chomsky, citando a otro catedrático del MIT: "No importa lo que se cubre (durante el semestre), sino lo que se descubre". Descubrir, crear, educación permanente, autonomía, imaginación, 
reflexión: parecieran ser los objetivos que una educación de calidad debiera tener en el siglo XXI.

En el texto de Torres, volvemos al tema de la ilustración, pues se señalan tres funciones de la educación: una función integradora, favorecer los niveles de igualdad y estimular el desarrollo de cada ser humano. Se debe tener como objetivo claro el lograr personas autónomas, responsables y solidarias; que exista un desarrollo de los estudiantes para que se integren a la sociedad y la hagan progresar; además de potenciar el desarrollo cognitivo, emocional, estético y físico para alcanzar la realización personal. En otras palabras, señala el autor:

La educación de las ciudadanas y ciudadanos para una sociedad democrática es necesario llevarla a cabo en el marco de instituciones en las que sus estructuras democráticas permitan la planificación y desarrollo de experiencias de enseñanza y aprendizaje dirigidas a promover y ejercitar la capacidad de tomar decisiones de modo reflexivo y de comprometerse en la realización de conductas responsables y efectivas, dentro de una filosofía de respeto y apoyo de los valores y procesos democráticos. Una educación crítica para una sociedad democrática implica que los alumnos lleguen a cuestionarse las interpretaciones de la realidad excesivamente homogéneas con las que se trabaja en el desarrollo de los currículos en las aulas (Torres, 1998: I49).

En palabras muy simples, se debiera educar para que el alumnociudadano piense con cierta autonomía, identidad e integralidad. Agreguemos a lo anterior, el carácter transformador, el ser agente de cambio para una sociedad más equitativa y equilibrada. Según Paulo Freire, la educación verdadera es "praxis, reflexión y acción del hombre sobre el mundo para transformarlo" (Freire, 1997: 7). No sólo debe responder a los requerimientos de la sociedad, sino también debe poder transformarla. Un agente activo, participativo, creativo.

Considerar la educación según la línea de Chomsky, Freire, Naranjo o Torres implica que no sólo se revisen los aspectos de la sociedad antes expuestos, sino también el cómo realizamos nuestras prácticas pedagógicas. Si revisamos el currículo nacional, lo que se propone 
no es muy distinto a lo que acá concluimos, de hecho la LEGE (Ley General de Educación) en su artículo II, dice:

La educación es el proceso de aprendizaje permanente que abarca las distintas etapas de la vida de las personas y que tiene como finalidad alcanzar su desarrollo espiritual, ético, moral, afectivo, intelectual, artístico y físico, mediante la transmisión y el cultivo de valores, conocimientos y destrezas. Se enmarca en el respeto y valoración de los derechos humanos y de las libertades fundamentales, de la diversidad multicultural y de la paz, y de nuestra identidad nacional, capacitando a las personas para conducir su vida en forma plena, para convivir y participar en forma responsable, tolerante, solidaria, democrática y activa en la comunidad, y para trabajar y contribuir al desarrollo del país (MINEDUC, 2009).

Por lo tanto, si en lo oficial ya se declaran elementos que apuntan a lo defendido en estas páginas, ¿cuáles son los obstáculos que tienen nuestra educación en crisis? ¿Cuál es el eslabón en la cadena educativa que ha llevado al ciudadano a transformarse en un ser que vive pasivamente el "tener" y el simulacro? Probablemente la respuesta la encontremos en cómo los "ingenieros del consenso democrático" interpretan, planifican e implementan, por ejemplo, el artículo II, pues claramente, si ponemos sobre la mesa el concepto de "contribuir al desarrollo del país", los significados que le atribuyamos serán sumamente disímiles, según lo enfoquemos desde el "ser" o desde el "tener".

En la práctica del día a día, qué lejos estamos de concepciones de la educación que se maticen con la idea de libertad o reflexión. Qué lejos de lo expuesto por Victoria Peralta (2006: 27):

Uno de los propósitos básicos de la educación es favorecer el desarrollo integral y pleno de los educandos, de acuerdo a sus potencialidades, de manera que puedan vivir plenamente su etapa presente, y afrontar con éxito las siguientes, tanto en beneficio propio, como de la sociedad a la que pertenecen. Específicamente, este objetivo se traduciría en que los niños, los jóvenes y los adultos se desenvuelvan en forma adecuada 
en su medio, teniendo las competencias necesarias para ello y, a la vez, la preparación suficiente para comprender y participar en otros ambientes.

Y, por último, qué lejos de lo señalado por Lucini (I994: 23):

La escuela, y, dentro de ella, toda la comunidad educativa, en la perspectiva que acabamos de plantear, necesita, hoy en día, adquirir un compromiso con el renacer de la nueva humanidad y con la nueva forma de habitar el planeta que nos está demandando la realidad y la problemática social; un compromiso que ha de traducirse en la transformación de sus proyectos educativos y curriculares en proyectos de humanización impregnados de valores y de principios morales. Proyectos en los que, a partir de un análisis crítico con lo establecido, los alumnos y las alumnas puedan diseñar horizontes y alternativas personales en el arte de aprender a vivir y de humanizar el mundo con la esperanza de poder transformarlo.

\section{Conclusiones}

El estado actual de la educación en Chile, en lo declarado, pareciera corresponderse con un modelo de educación que apunta a la ilustración según lo expuesto por Chomsky. No obstante, en la práctica, durante el desarrollo real de los currículos nacionales e institucionales, prevalece una mixtura con lo oculto, una correspondencia con lineamientos de adoctrinamiento de los educandos para responder a las necesidades competitivas del mercado -que han olvidado el ser, para centrarse en el tener- $y$ a un adormecimiento del cuestionamiento, la crítica y la reflexión.

De tal manera, se han analizado las características de la sociedad actual, para buscar la pertinencia de continuar con una educación que responda a las necesidades actuales o, de plano, plantear la búsqueda de una educación que tenga la potestad de transformar la sociedad. Según autores como Baudrillard, Debord y Zizek, la sociedad vive en una especie de simulación de la realidad, carente de total humanismo, y que ha aceptado la irrupción de la economía neoliberal como si fuese un hecho incuestionable. Se podría discutir o cuestionar todo 
lo demás, pero no la economía, porque se le ha deslindado de su carácter ideológico, por lo tanto, sin espacio de deliberación. En esta situación de la sociedad, surge la necesidad de desarrollar una educación que tenga por objetivo transformar el estado de las cosas.

En sintonía con esa última idea, autores como Peralta, Naranjo, Freire, Lucini, Chomsky y Torres dan señales de cómo debiera ser dicha educación:

- Permitir que las personas aprendan por sí mismas. Autonomía, reflexión, formación permanente.

- Cuestionamiento de las verdades absolutas, especialmente las doctrinas. Desarrollo de la crítica y la autocrítica.

- Fortalecimiento de la imaginación.

- Trabajo cooperativo.

- Identidad, integralidad.

- Un claro propósito de transformación de la sociedad.

En síntesis, con esos objetivos de la educación sumamente claros, no sólo apuntaríamos a no responder a los lineamientos de ofertademanda de la sociedad actual, sino que se crearían nuevas vías de desarrollo, teniendo en el horizonte muy presentes los valores humanistas, de comunión social, creatividad y reflexión; en equilibrio con el medio ambiente y siempre direccionando las intenciones hacia un desarrollo integral y exponencial del ser humano, no en el tener, sino en el ser; no constituyéndose esta reflexión sólo como una crítica hacia el sistema neoliberal, sino comprendiendo el sistema neoliberal como opción, no como imposición sin matices.

De este modo, finalmente, se oyen cada vez con mayor resonancia las palabras de Humberto Maturana: "Si la educación en Chile no lleva a los jóvenes chilenos a la responsabilidad y libertad de ser cocreadores del mundo en que viven porque limita la reflexión, la educación en Chile no sirve ni a Chile ni a los chilenos." (Maturana. 1997: 35). 


\section{Referencias bibliográficas}

Atria, F. (2007). Mercado y ciudadanía en la educación. Santiago, Chile: Editorial Flandes Indiano.

Baudrillard, J. (1993). Cultura y simulacro. Barcelona, España: Kairós.

Chomsky, N. (2012). Los objetivos de la educación. Londres, Inglaterra: Conferencia Aprendizaje sin Fronteras (audio). Fecha de consulta 25 de marzo 2013. Disponible en: http://redaccion.hypotheses.org/397

Chomsky, N. (2003). Piratas y emperadores. Barcelona, España: Ediciones B, S.A.

Chomsky, N. (I994). Política y cultura a finales del siglo XX. Un panorama de las actuales tendencias. España: Ariel SA.

Debord, G. (1967). La sociedad del espectáculo. París, Francia: Champ libre. (Traducción del Archivo Situacionista Hispano, 1998).

Freire, P. (1997). La educación como práctica de la libertad. México D.F., México: Siglo XXI editores, 45 edición.

Lucini, F. (1994). Temas transversales y áreas curriculares. Madrid, España: Grupo Anaya.

Maturana, H. (1997). Emociones y lenguaje en educación y política. Santiago, Chile: Ediciones Dolmen, novena edición.

Mayol, A.; Araya, J.; Azócar, C. \&Azócar, C. (20I I). 7 fenómenos sobre educación y desigualdad en Chile. Santiago, Chile: CIES Universidad de Chile.

MINEDUC (201 I). Resultados PISA 2009, Chile. Santiago, Chile: Autor.

MINEDUC (2009). Ley General de educación. Santiago, Chile: Autor.

Naranjo, C. (2007). Cambiar la educación para cambiar el mundo. Santiago, Chile: Editorial Cuarto Propio.

Peralta, V. (2006). Currículos educacionales en América Latina su pertinencia cultural. Santiago, Chile: Editorial Andrés Bello, segunda edición.

Torres, J. (1998). El currículum oculto. Madrid, España: Ediciones Morata SL.

Zizek, S. (1999). Dije economía política, estúpido. Extraído de The Ticklish Subject (Londres: Verso), publicado por Página/30 Nro. I18, mayo 2000. 